\title{
INDEX for Volume 107
}

S. Alesker \& A. Bernig, Convolution of valuations on manifolds.

L. Ambrozio, On static three-manifolds with positive scalar cur-

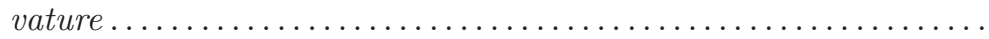

S. T. Becker-Kahn, Transverse singularities of minimal two-valued graphs in arbitrary codimension......................... 241

D. Chen, Degenerations of Abelian differentials ............... 395

T. C. Collins \& G. Székelyhidi, Convergence of the J-flow on toric

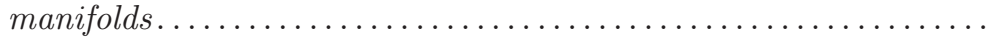

S. Donaldson \& S. Sun, Gromov-Hausdorff limits of Kähler manifolds and algebraic geometry, $I I \ldots \ldots \ldots \ldots \ldots \ldots \ldots \ldots \ldots$.

A. García-Raboso, A twisted nonabelian Hodge correspondence..

C.-Y. Hsiao \& G. Marinescu, On the singularities of the Szego"

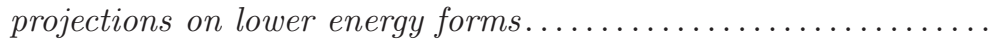

T. Kuwagaki, The nonequivariant coherent-constructible correspondence for toric surfaces.............................

D. Maximo, I. Nunes, \& G. Smith, Free boundary minimal annuli

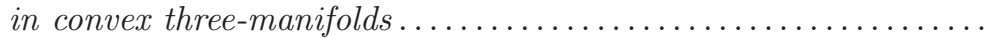

M. Mayer \& C. B. Ndiaye, Barycenter technique and the Riemann

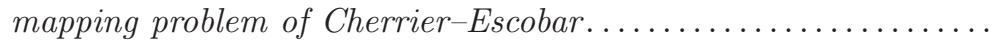

G. Meigniez, Regularization and minimization of codimension-one

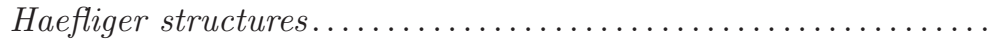

N. Nadirashvili \& Y. Sire, soperimetric inequality for the third eigenvalue of the Laplace-Beltrami operator on $\mathbb{S}^{2} \ldots \ldots \ldots . .$.

V. Tosatti \& X. Yang, An extension of a theorem of Wu-Yau... 